\title{
FORMATION OF THE SYSTEM OF CORONASTATISTICS INDICATORS
}

\section{SIMON GELASHVILI}

\author{
Doctor of Economic Sciences, Professor, \\ Academician of Georgia Economic Sciences Academy \\ Ivane Javakhishvili Tbilisi State \\ University, Georgia \\ simon.gelashvili@tsu.ge
}

Abstract. The paper discusses Coronastatistics as a content of complex cognition, which is characterized by many aspects; it reflects not only the health care issues of the population, but also the issues of the economy: A system of their characteristic statistical indicators and its constituent blocks is proposed. Each of them gives a fairly wide range of both general and specific indicators. It is clear that health statistics indicators are particularly widespread, quantitatively characterizing the total number of Coronavirus-infected populations as well as their distribution by area, source of disease, sex, age, and other signs.

The economic bloc presents statistical indicators, that characterize the impact of COVID-19 on the economic and business sectors and the consequences of its impact in the short, as well as medium and long term. Both, macro and microeconomic statistical measures are provided, as well as indicators for determining the size and range of reactions of small, medium and large businesses.

The COVID-19 pandemic has more or less spread to almost every country, and it has had a significant unintended negative impact on all areas of public life, especially the economy and human social life. This impact is multifaceted and requires complex quantitative and qualitative research. Appropriate methodoland methodology, including statistics, are necessary for such research.

Coronastatistics has a broad, complex content and includes not only quantitative indicators of the spread of the Coronavirus, but also the consequences of its impact on human health and social life, as well as economic, environmental, demographic, business and other areas. The system of Coronastatistics consists of many subsystems, or blocks. First of all, the groups of general and special indicators should be distinguished, which are created according to each field. The grouping is based on various essential traits such as field, area, demographic (biological) characteristics and many more. The calculated indicators will be represented as absolute and relative, as well as average, in the form of variation and indices. In addition, appropriate time series and correlation analysis indices should be used to quantify the impact of the COVID-19 pandemic.

For the first time in world history, the main cause of the current local, regional or global economic crisis is not directly in the economy or its management, but due to the impact of another, very strong exogenous factor - the COVID-19 pandemic. In such conditions, the need to expand the functions of the government is on the agenda. The state also takes the risk in managing the national economy, but it is somewhat different (Papava V., 2020: 138).

It should also be noted that it is very difficult to accurately quantify the impact of the COVID-19 pandemic on the economy and business, both globally and locally (depending on the country), in the short or medium term. This begs the question: should economic and business change forecasts be calculated? The correct answer is: Of course, such predictions are needed. But they must be reliable enough. "In economics and business, forecasting serves as a compass that shows you where to go." (Gelashvili, S., 2019: 13). Therefore, in this case, it is more appropriate to calculate the interval statistical forecasts on the principle of collaborative forecasting, especially in the field of business, through which it is possible to carry out an operational response to specific market processes (Gelashvili, S., 2017: 259).

The article presents the systems of coronastatics indicators developed by the author in the fields of economics and healthcare, which allows for a complex quantitative analysis and the development of appropriate recommendations.

During the COVID-19 pandemic, the managerial and preventive functions of the statistics were particularly evident. Without complete and reliable information and proper indicators, it is impossible to make sound and optimal decisions at all hierarchical levels and under any conditions, including pandemics or normal living conditions.

The complex system of Coronastatistics indicators, proposed by the author, allows typological, structural and analytical grouping of data on COVID-19, which is an important condition for conducting relevant qualitative and quantitative analysis.

KEYWORDS: CORONASTATISTICS, COMPLEX SYSTEM OF INDICATORS, GENERAL INDICATORS, SPECIFIC INDICATORS, GROUPING.

For citation: Gelashvili, S., (2020). Formation of the System of Coronastatistics Indicators. Globalization and Business, 10. 63-69. (In Georgian) https://doi.org/10.35945/gb.2020.10.007 


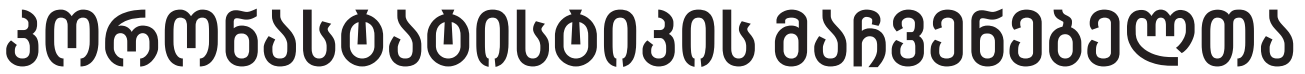

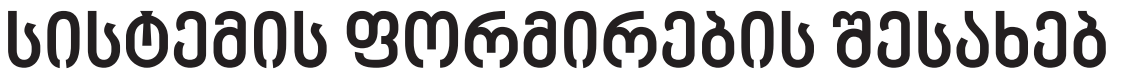

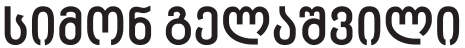

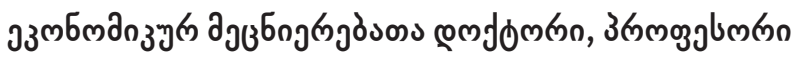

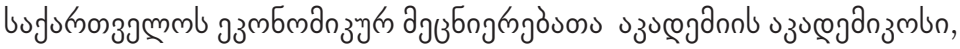

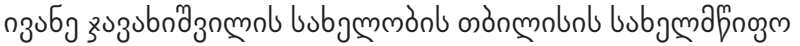

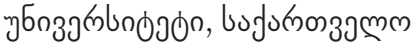 \\ simon.gelashvili@tsu.ge
}

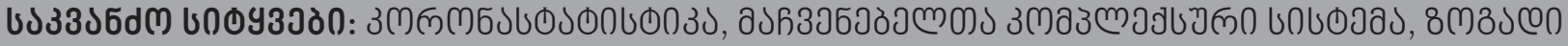

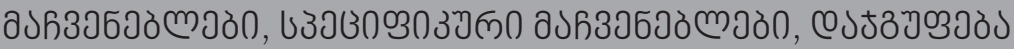

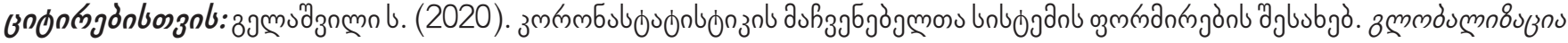
cou òn86jlo, №10, 33. 63-69. https://doi.org/10.35945/gb.2020.10.007

\section{ə0เ১3১ल0}

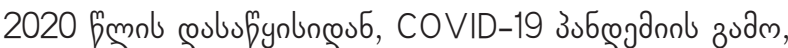

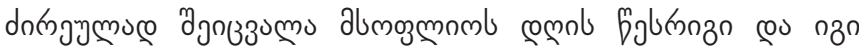

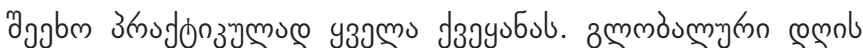

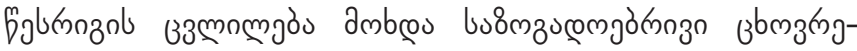

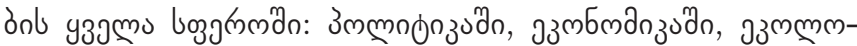

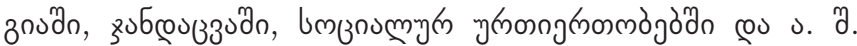

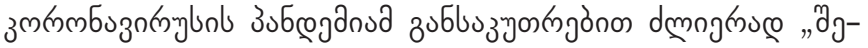

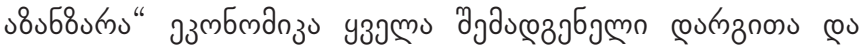

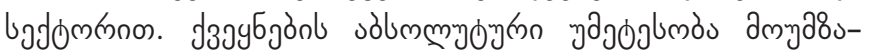

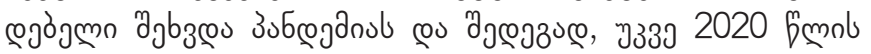

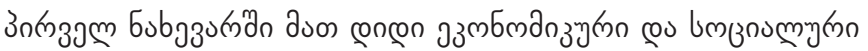

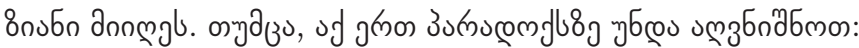

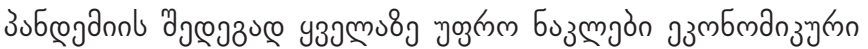

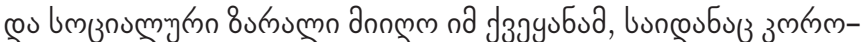

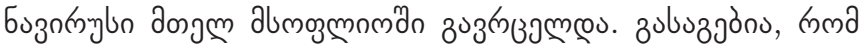

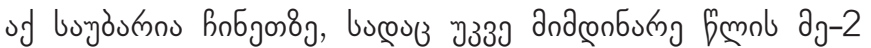

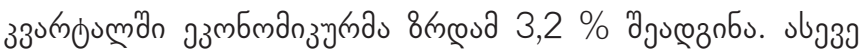

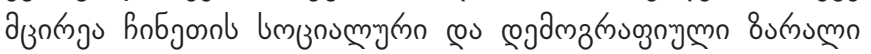

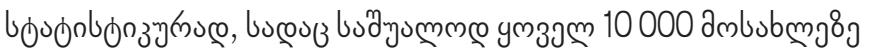

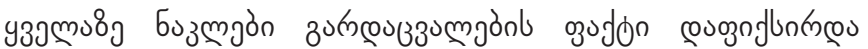

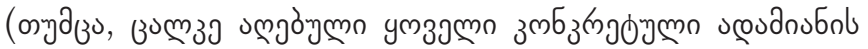

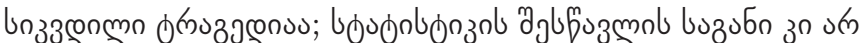

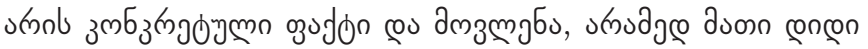

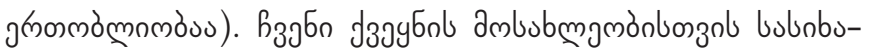

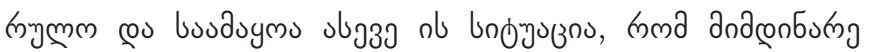

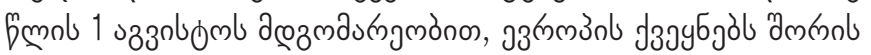

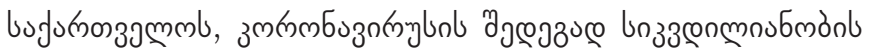

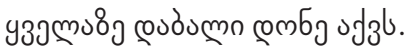

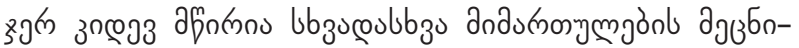

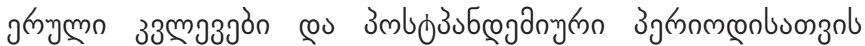

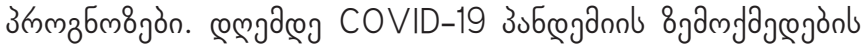

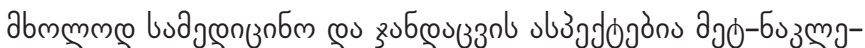

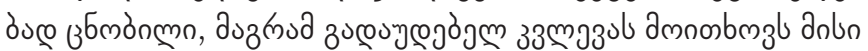

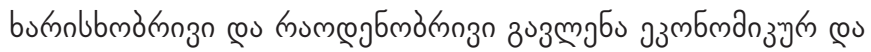

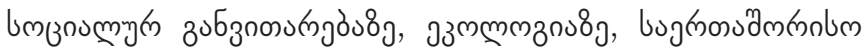

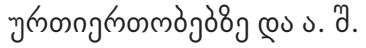

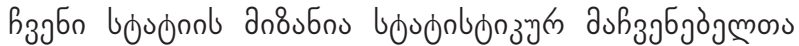

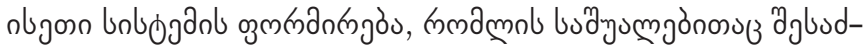

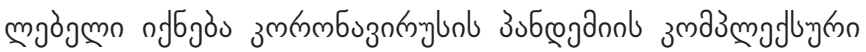

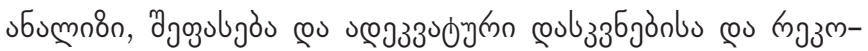

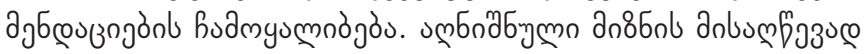

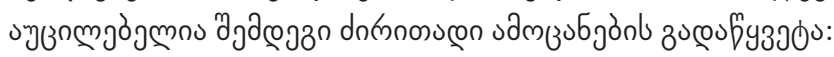

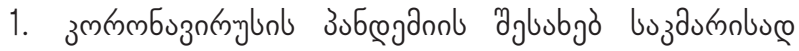

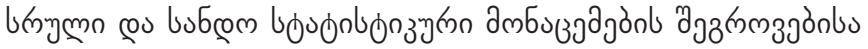

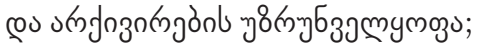

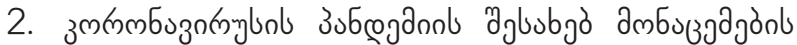

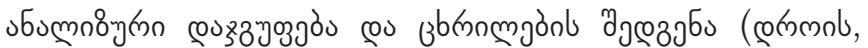

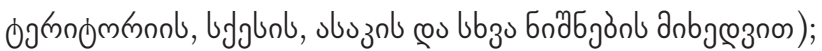

3. एовз

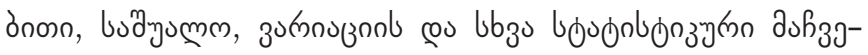

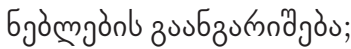

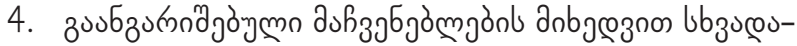

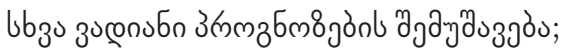

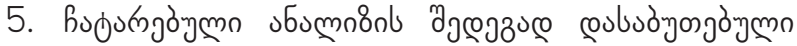
couluz

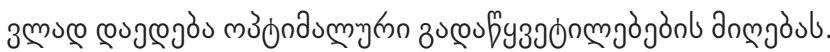

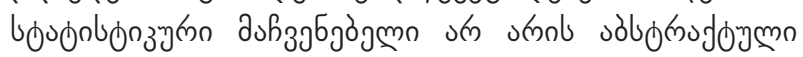

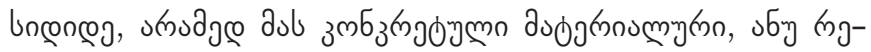

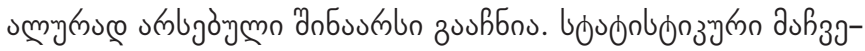

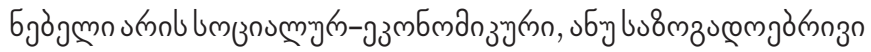




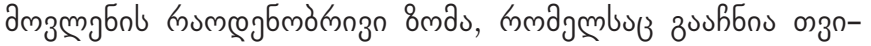

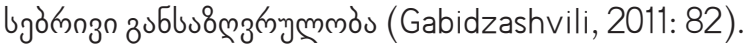

\section{domnosugo gobsumb}

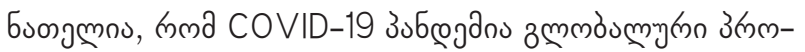

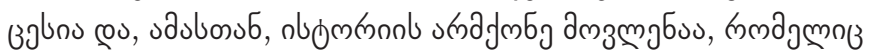

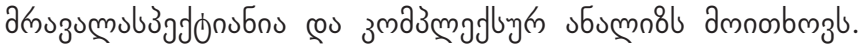

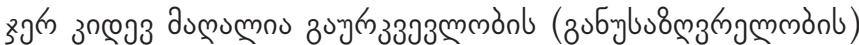

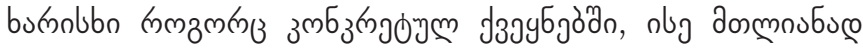

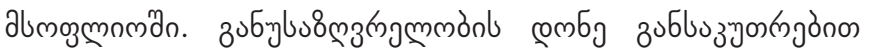

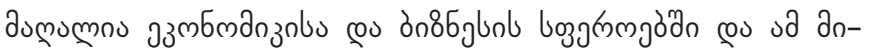

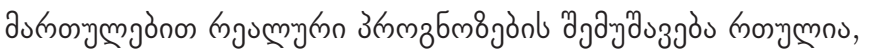

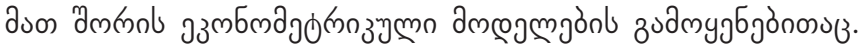

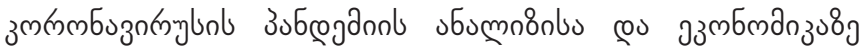

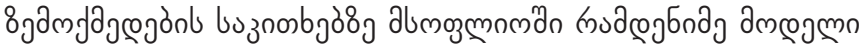

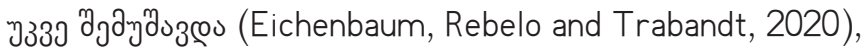

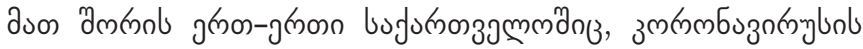

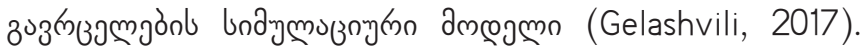

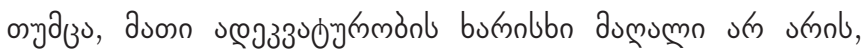

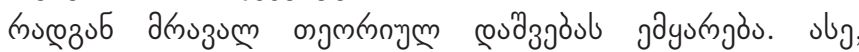

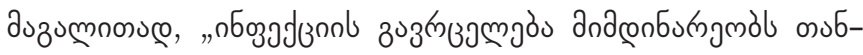

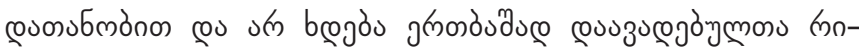

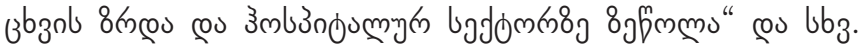

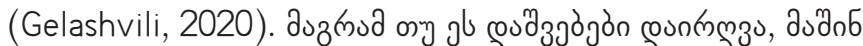

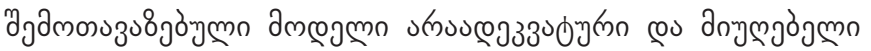
กffjoso.

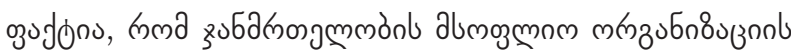

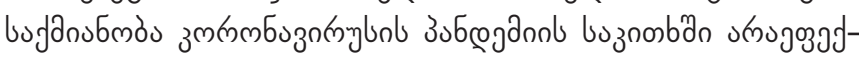

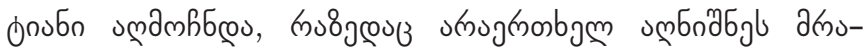

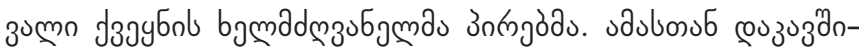

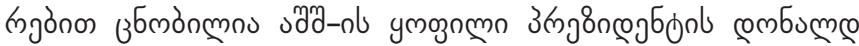

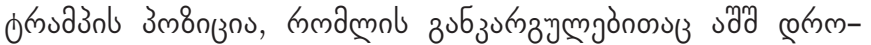

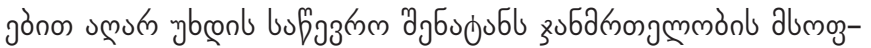

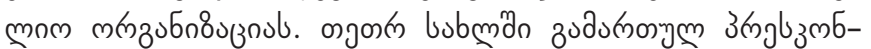

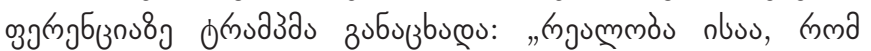

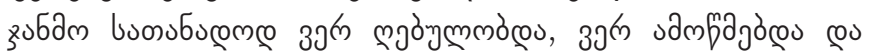

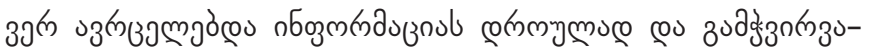

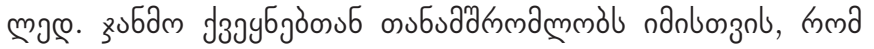

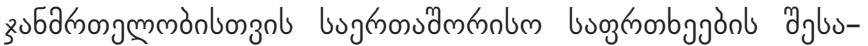

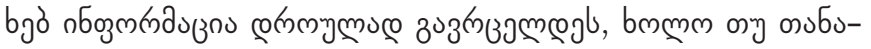

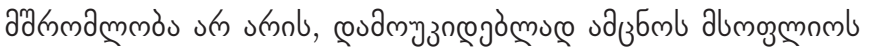
amabosumo

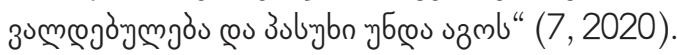

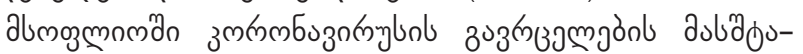

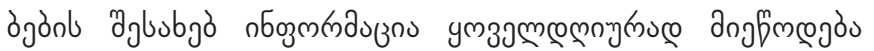

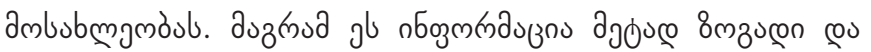

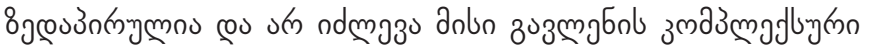

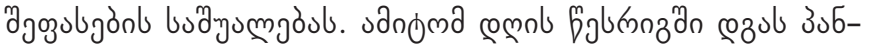

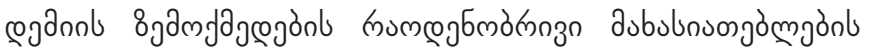

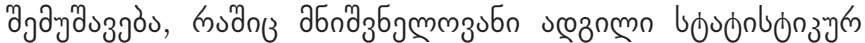

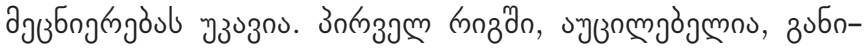

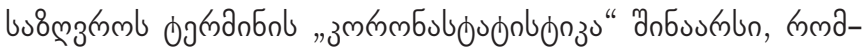

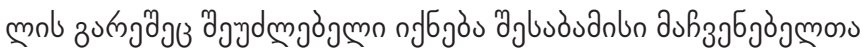

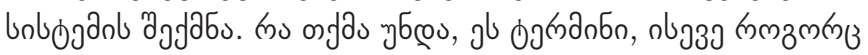

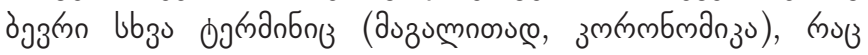

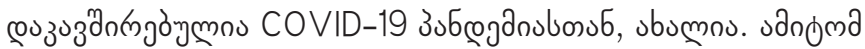

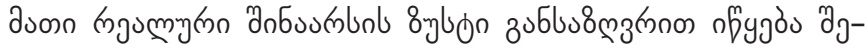

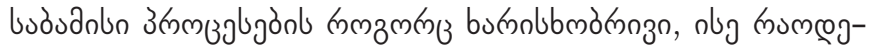

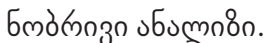

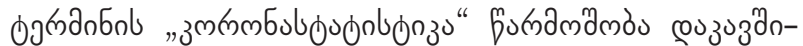

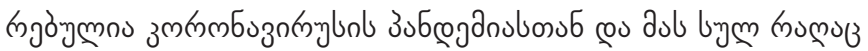

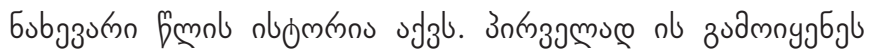

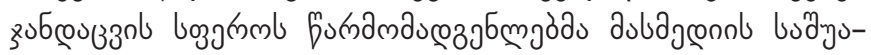

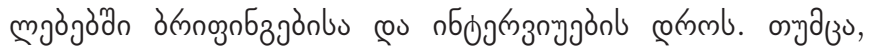

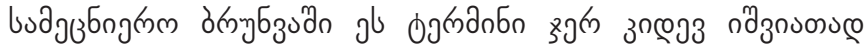

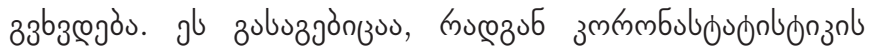

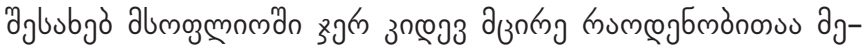

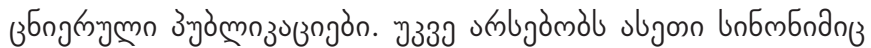

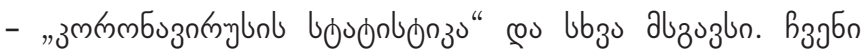

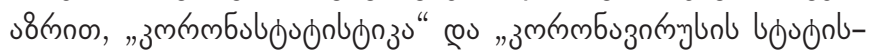

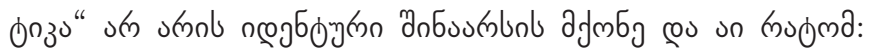

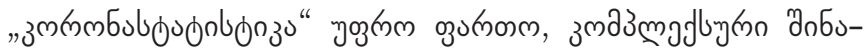

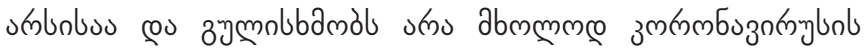

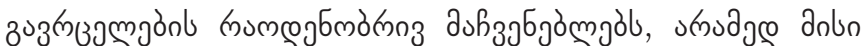

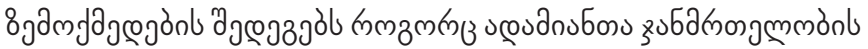

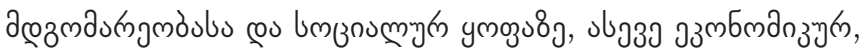

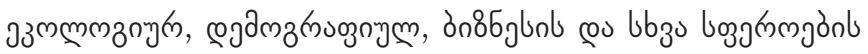

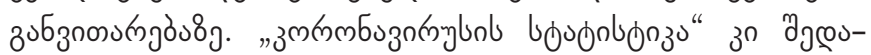

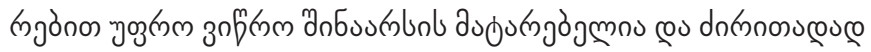
uмб

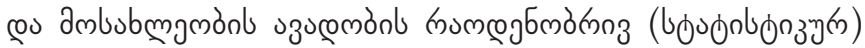

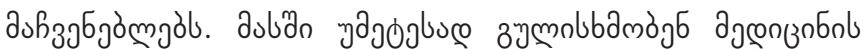

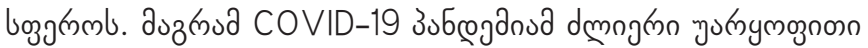

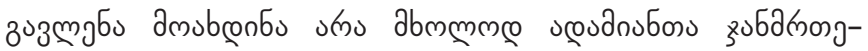

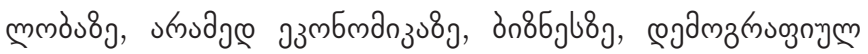

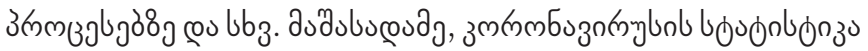

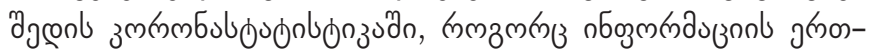

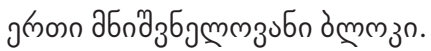

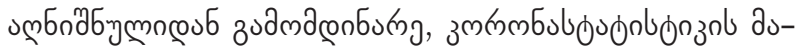

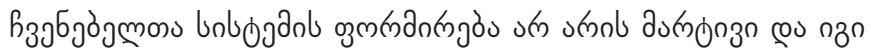

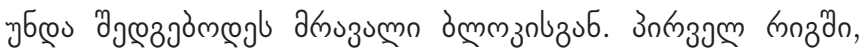

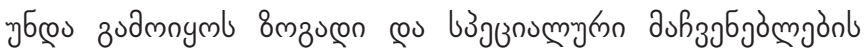

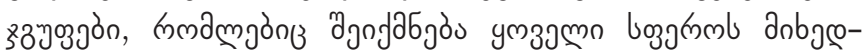

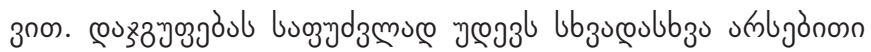

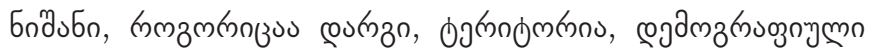

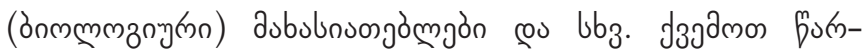

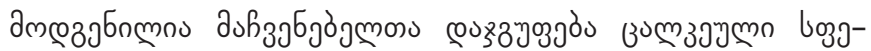

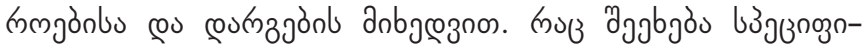

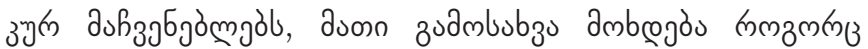




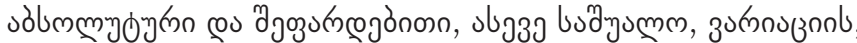

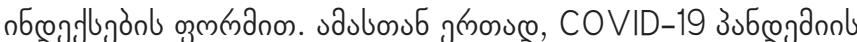

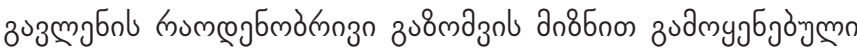

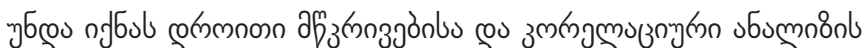

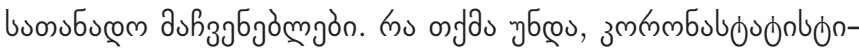

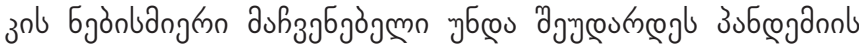

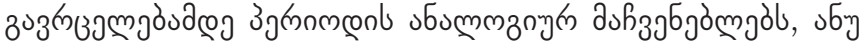

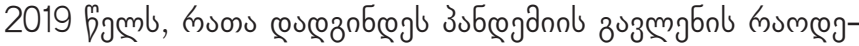

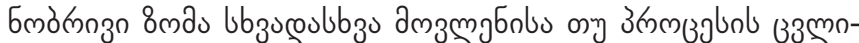
mgobog.

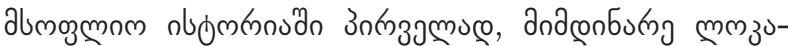

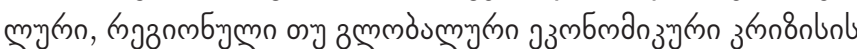

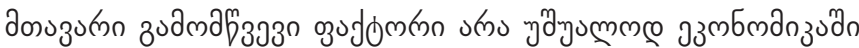

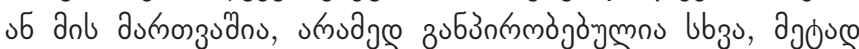

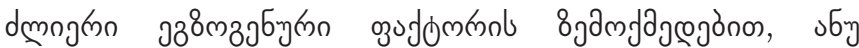

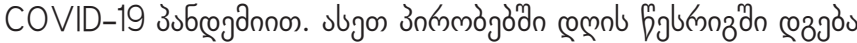

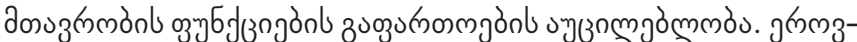

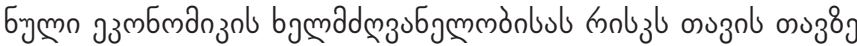

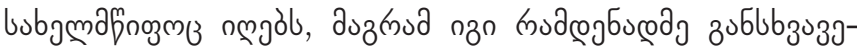
J'ymo bulnoonnlus (Papava, 2020: 138).

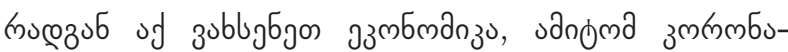

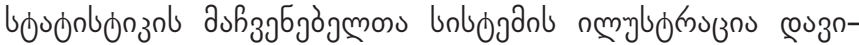

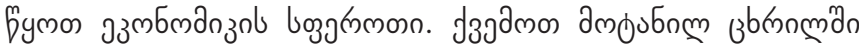

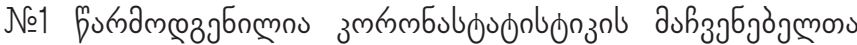
unlogas эзmбmanzoдn.

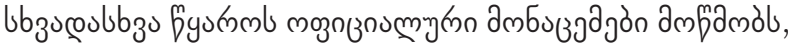

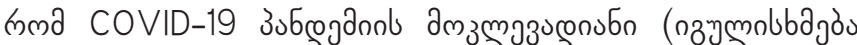

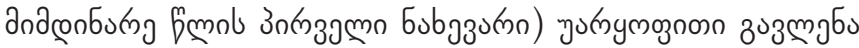

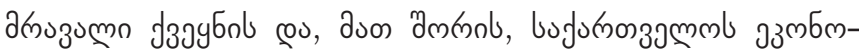

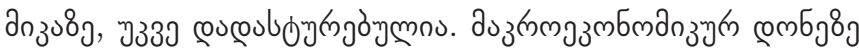

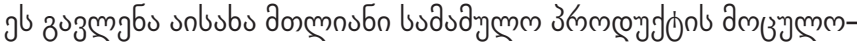

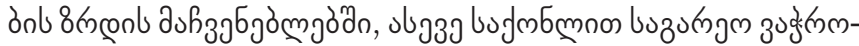

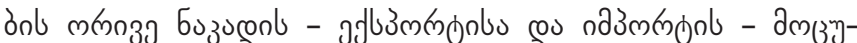

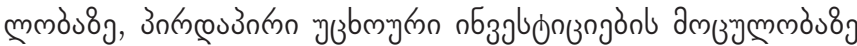

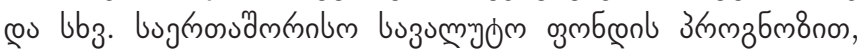

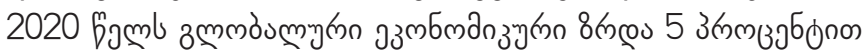

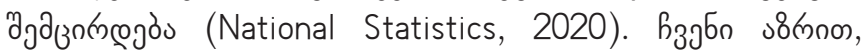

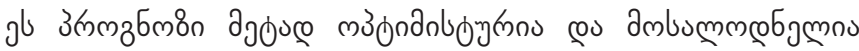

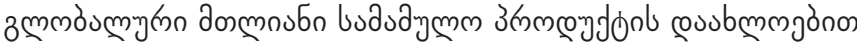

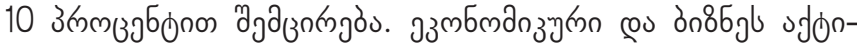

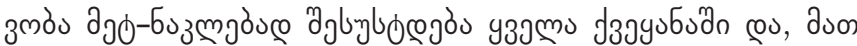

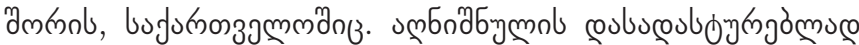

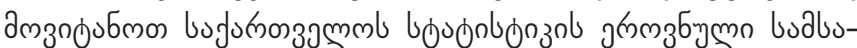

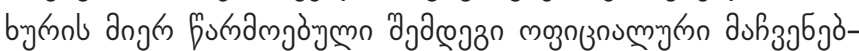

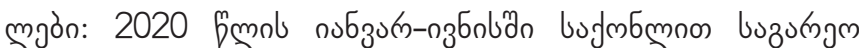

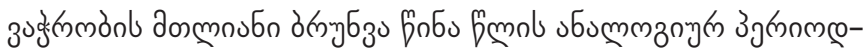

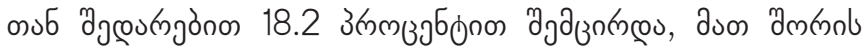

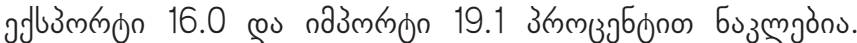

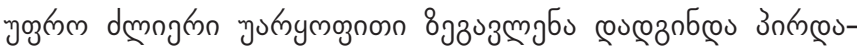

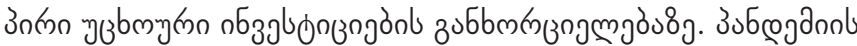

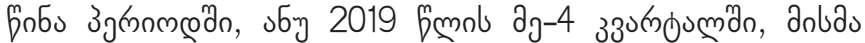

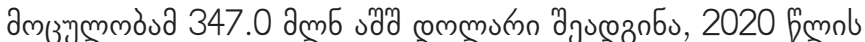

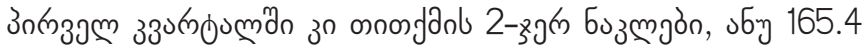
a

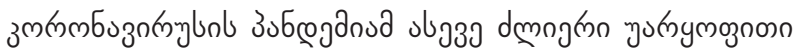

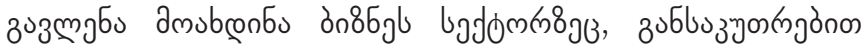

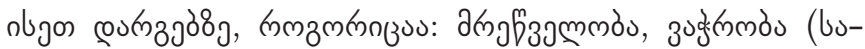

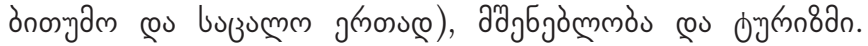

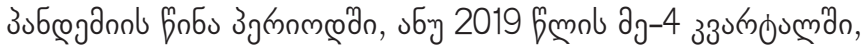

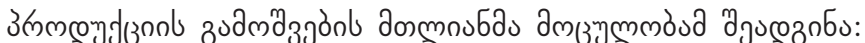
дங

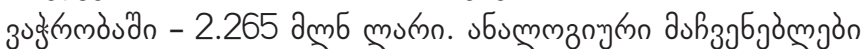

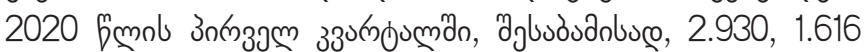

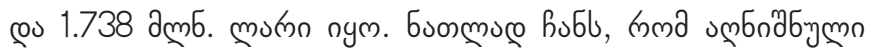

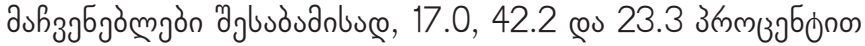

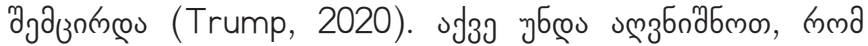

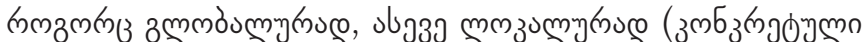

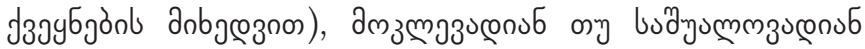

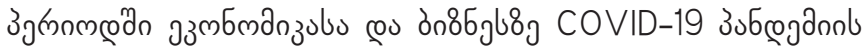

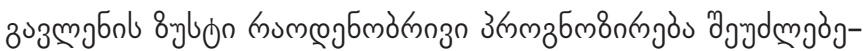

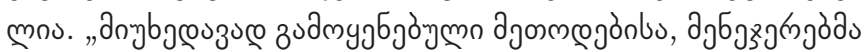
चб

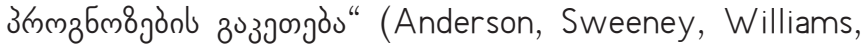

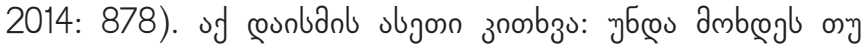

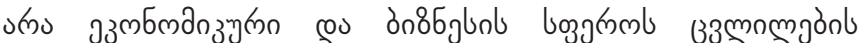

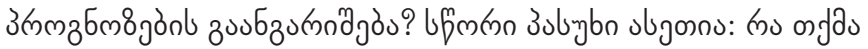

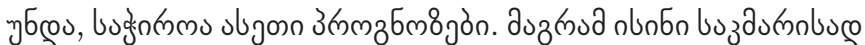

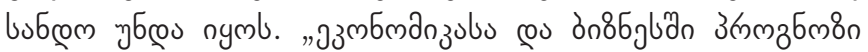

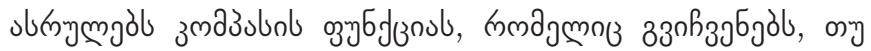

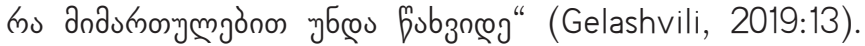

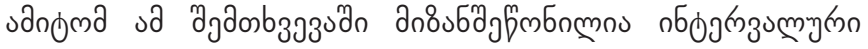

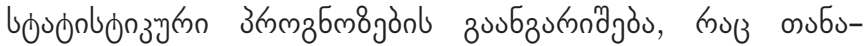

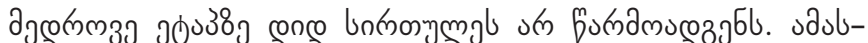

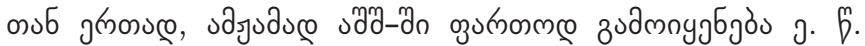

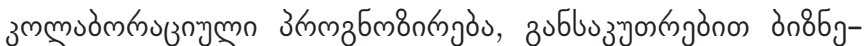

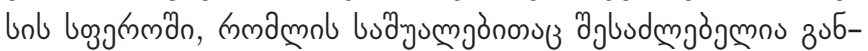

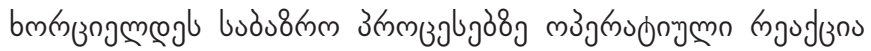
(Gelashvili, 2017:259).

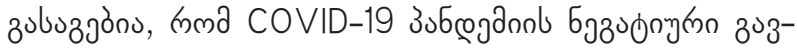

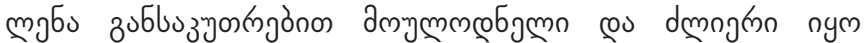

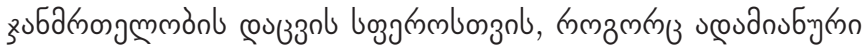

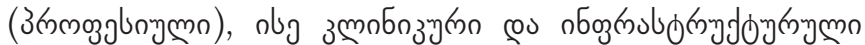

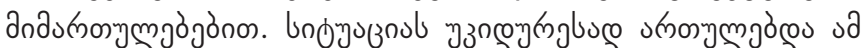

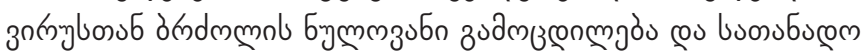

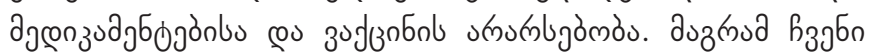

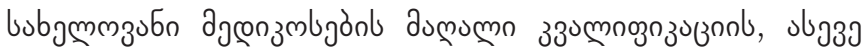

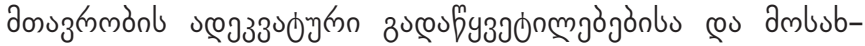

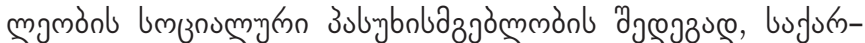

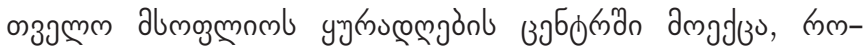

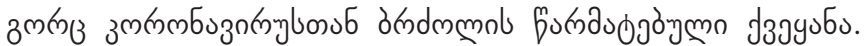




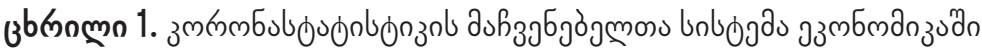

\begin{tabular}{|c|c|c|}
\hline ह̆mృdo & 2020 & 2020 \\
\hline logुलm & 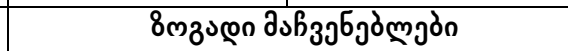 & 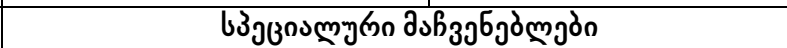 \\
\hline эзмбмдпзЈ & 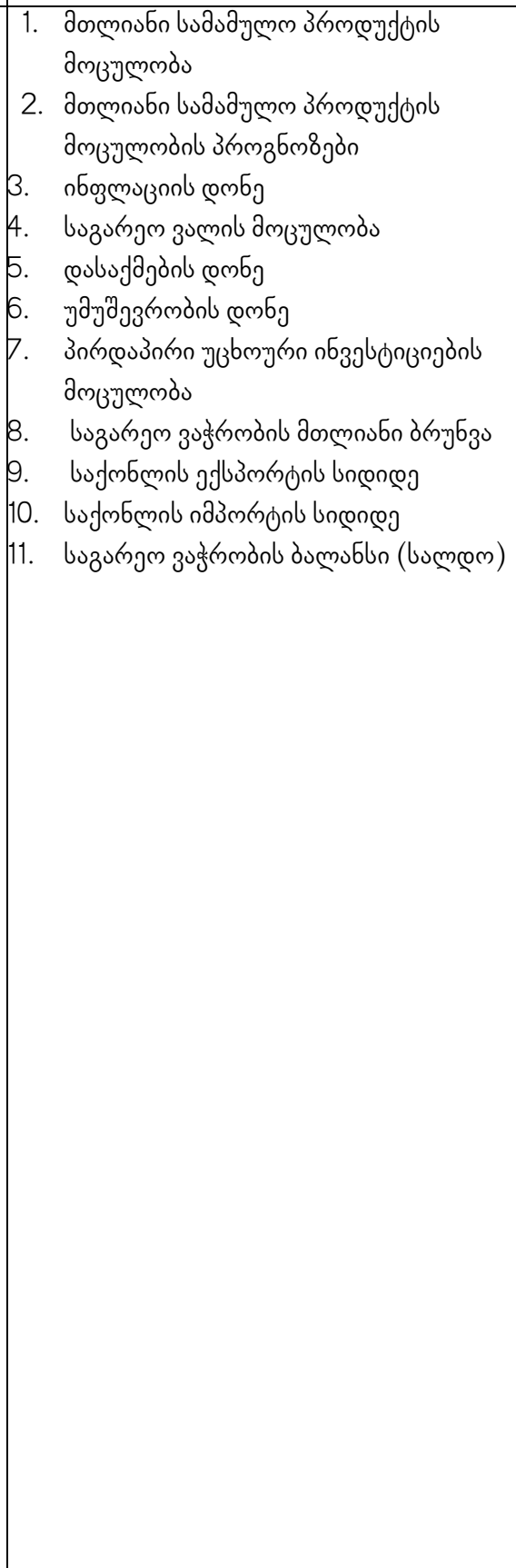 & 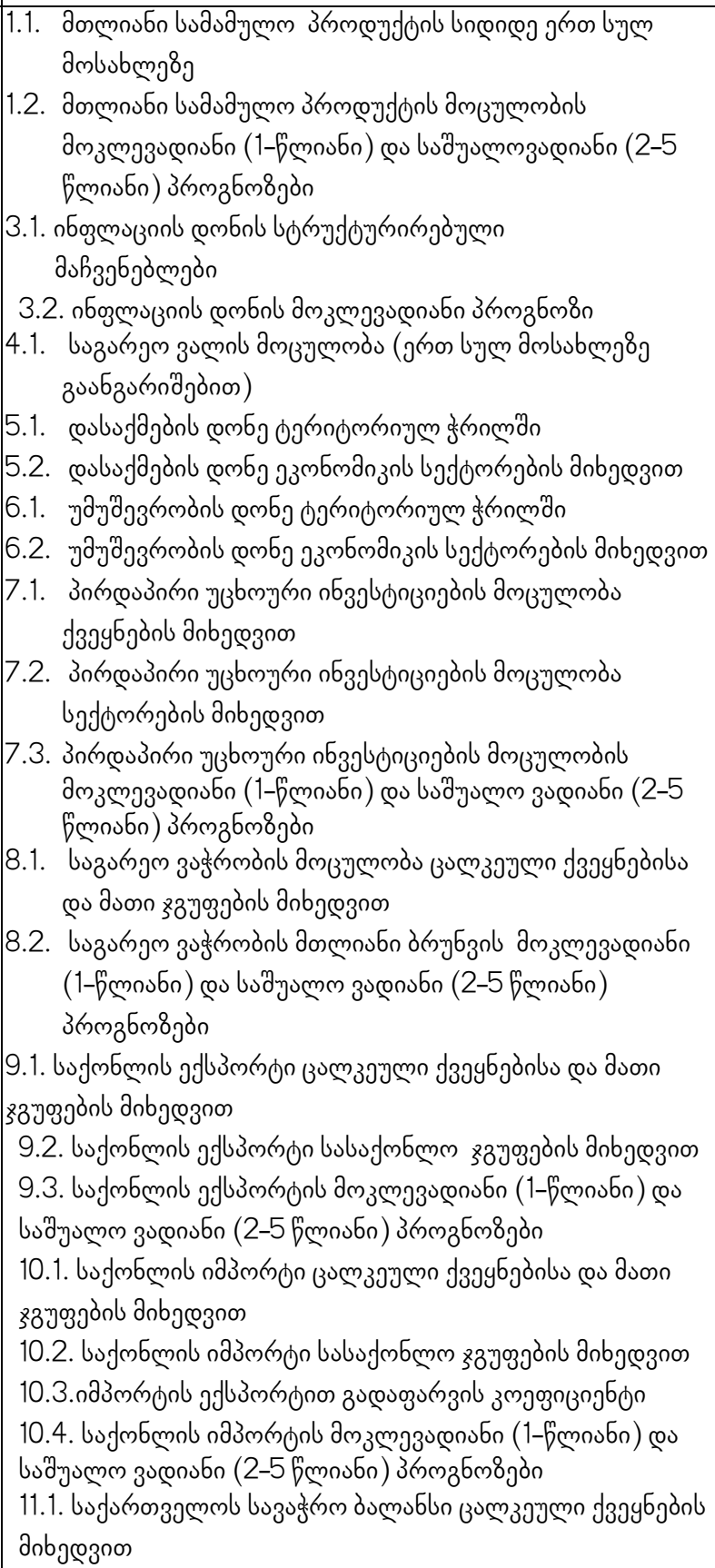 \\
\hline
\end{tabular}

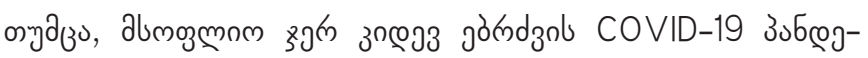

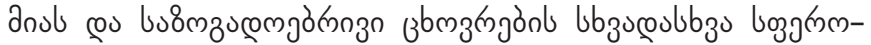

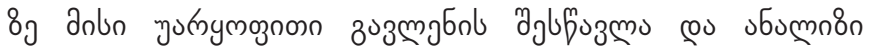

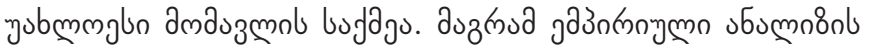

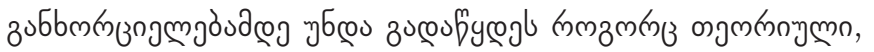

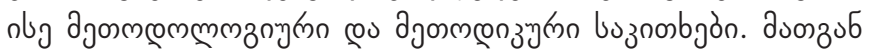

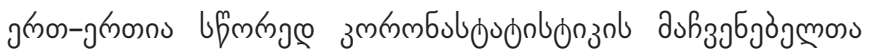

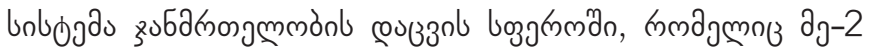

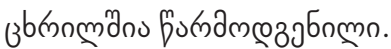

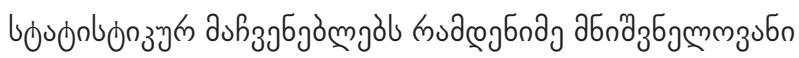

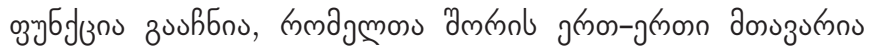

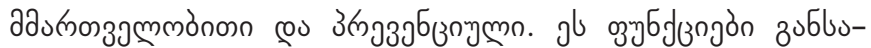

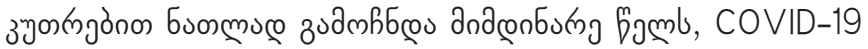

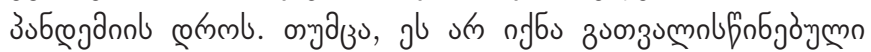

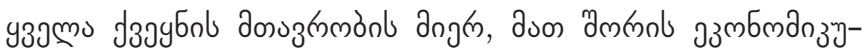

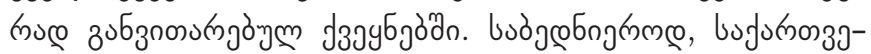

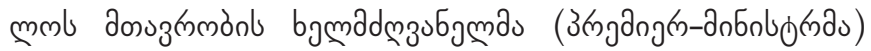

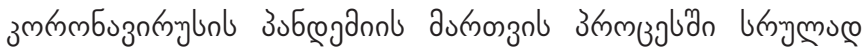




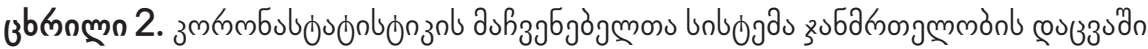

\begin{tabular}{|c|c|c|}
\hline bmgòn & 2020 & 2020 \\
\hline loggmm & 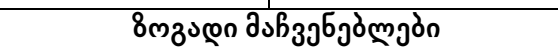 & 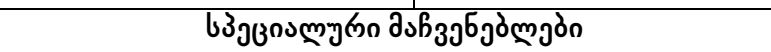 \\
\hline 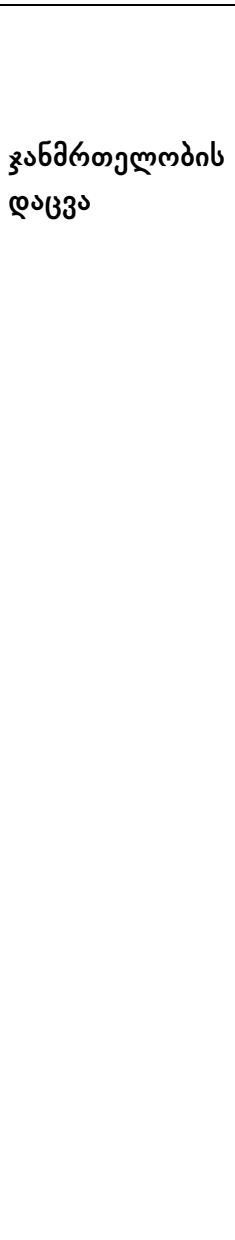 & 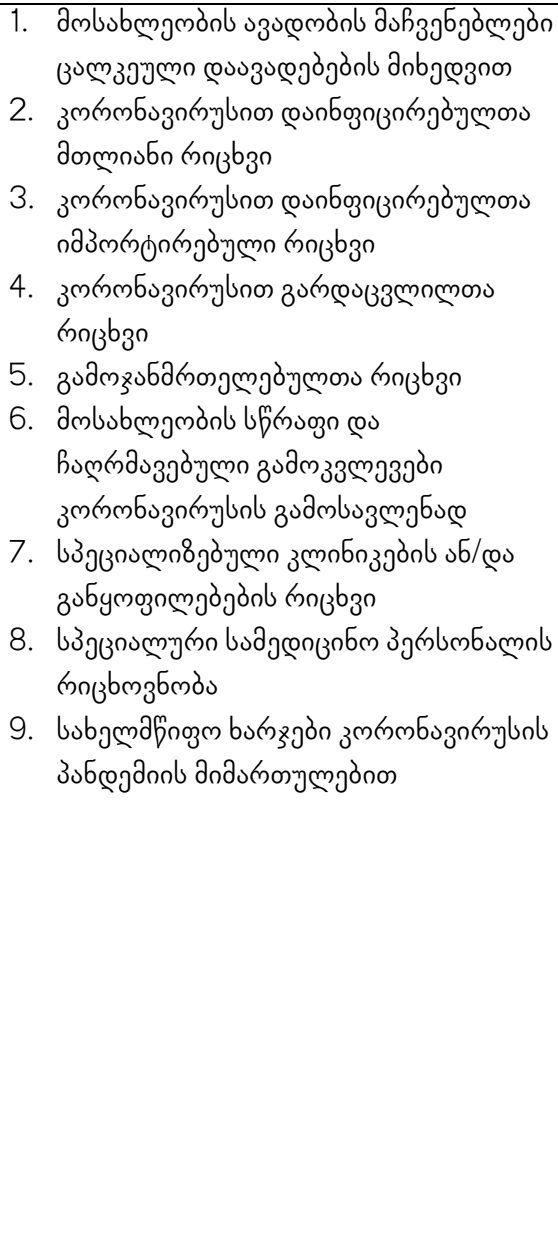 & 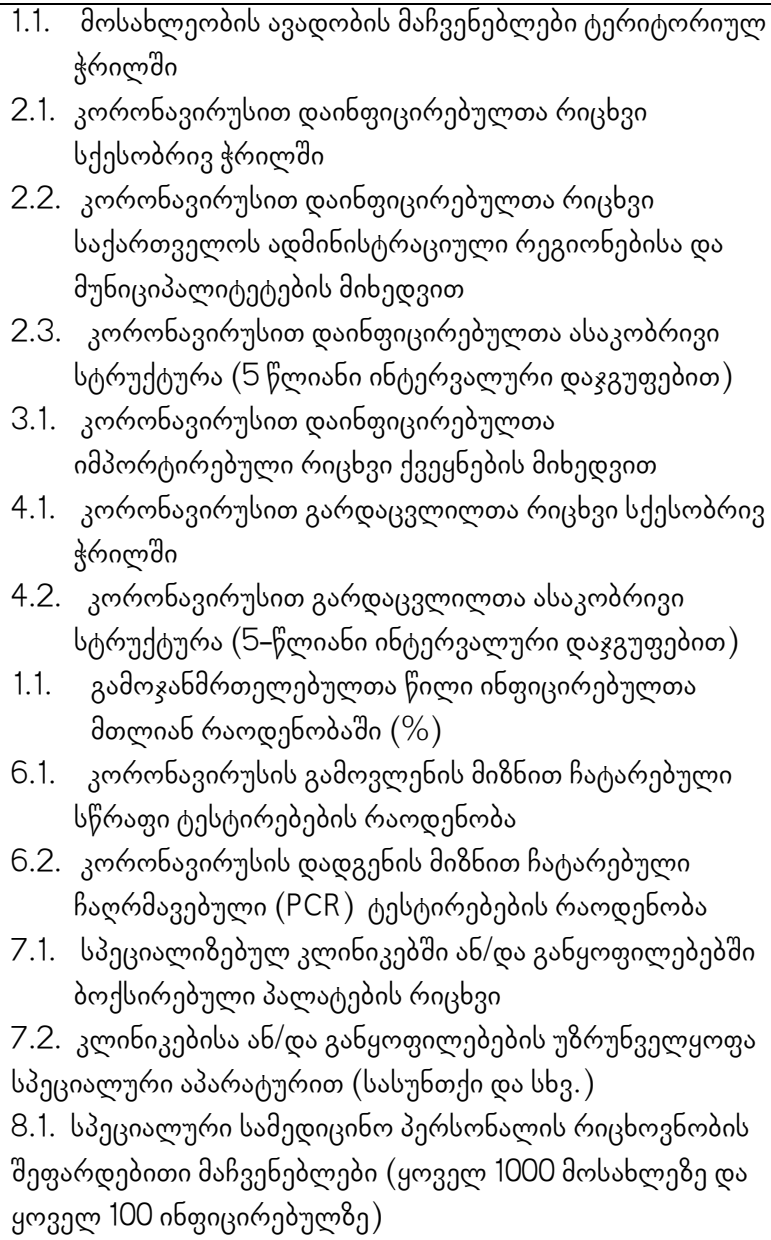 \\
\hline
\end{tabular}

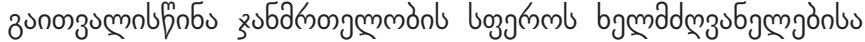

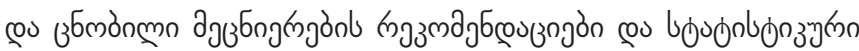

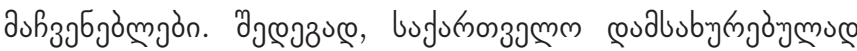

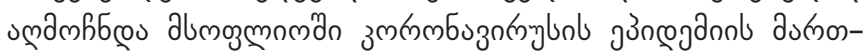

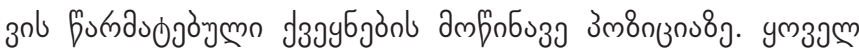

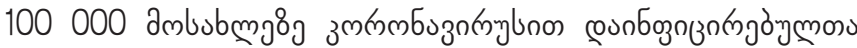

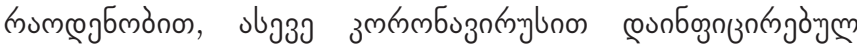

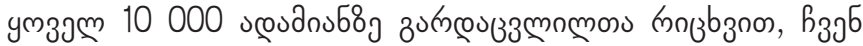

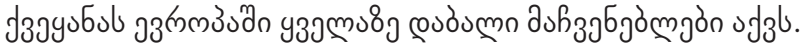

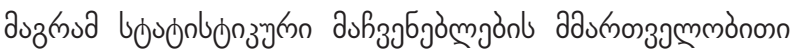

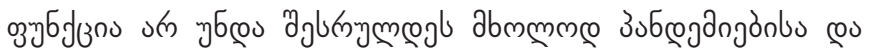

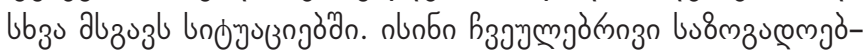

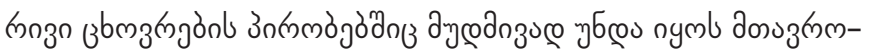

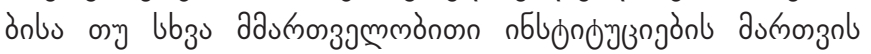

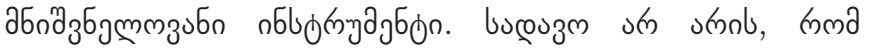

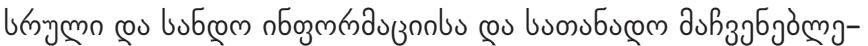

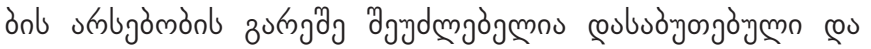

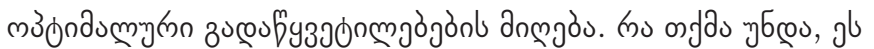

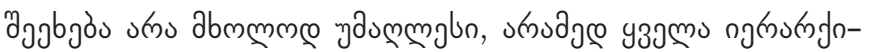

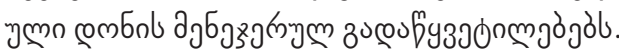

\section{@su336s}

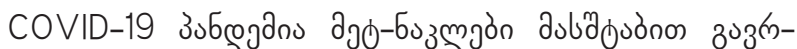

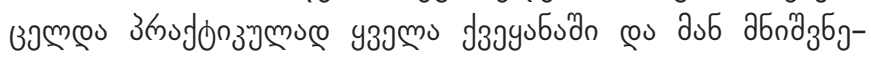

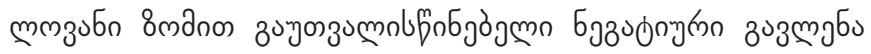

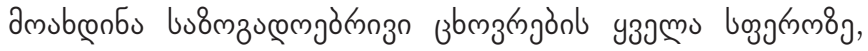
zubluzचулm

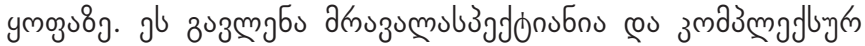

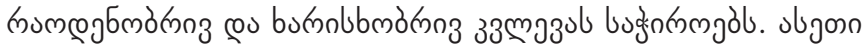

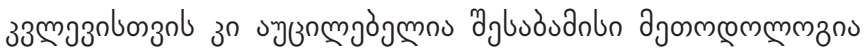
es дjommenzu.

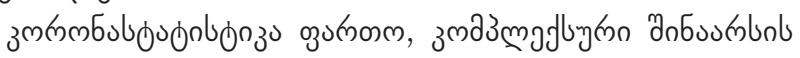

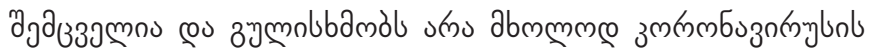

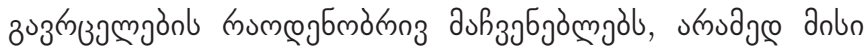

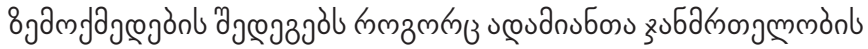

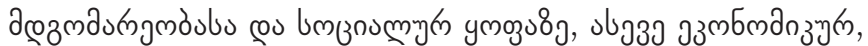

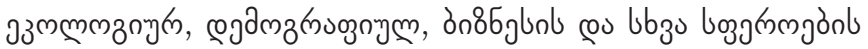

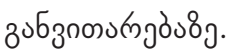

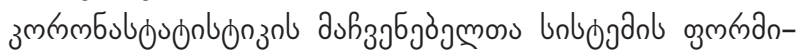

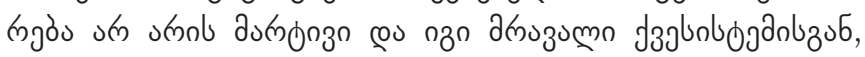




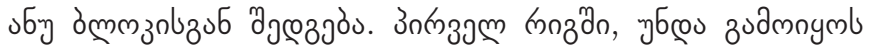

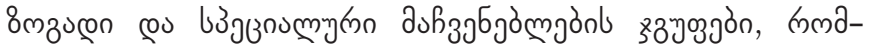

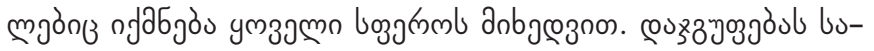

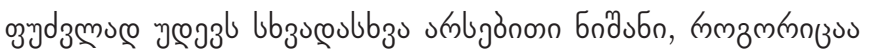

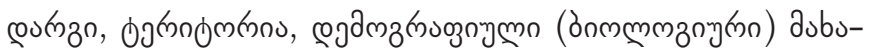

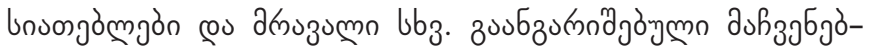

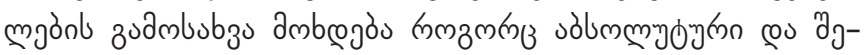

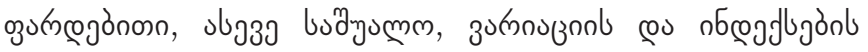
өुmरुमnon.

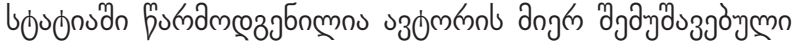

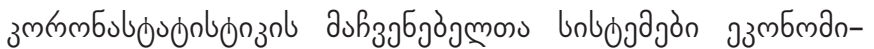

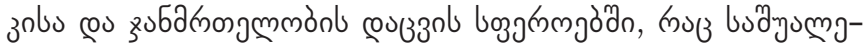

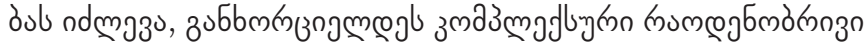

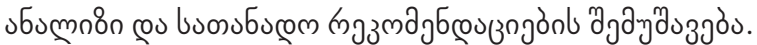

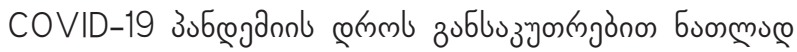

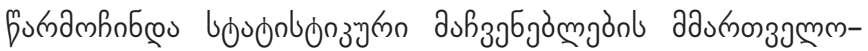

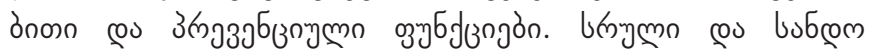

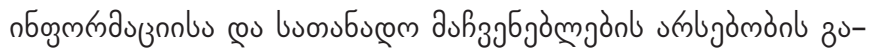

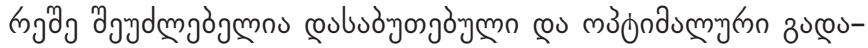

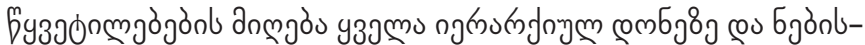

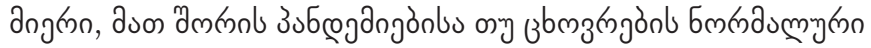

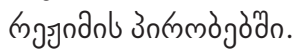

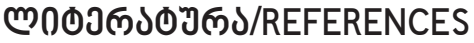

Anderson, D., Sweeney, D., Williams, Th. (2014). Statistics for Business and Economics. TSU Publishing, (in Georgian).

Eichenbaum, M., Rebelo S. and Trabandt M. (2020). The macroeconomics of epidemics. w26882. National Bureau of Economic Research.

Gabidzashvili, B. (2011). Statistics for Economics, Business and Management. Publishing - Universali. (in Georgian)

Gelashvili, S. (2019). Introduction to Statistical Forecasting. Publishing - Universali. (in Georgian)

Gelashvili, S. (2017). Statistical Forecasting in Economics and Business. I Book. Publishing - Meridiani. (in Georgian).

International Monetary Fund. (2020). World Economic Outlook Update: A Crisis Like No Other, An Uncertain Recovery. Washington, DC.

National Statistics Office of Georgia (2020). Statistical research - On Foreign Economic Activity. (in Georgian)

National Statistics Office of Georgia (2020). https://www.geostat.ge/ka/modules/ categories (in Georgian)

National Statistics Office of Georgia (2020). https://www.geostat.ge/ka/modules/ categories/191/pirdapiri-utskhouriinvestitsiebi (in Georgian)

Simulation model of Coronavirus spread - COVID-19 GE (2020). http://weg.ge/sites/ default/files/covid19_ge_anotacia.pdf (in Georgian)

Trump has cut funding for WHO (2020). https://pia.ge/ka/news/covid-19/trampma-janmos-daphinanseba-sheutskvita (in Georgian)

Papava, V. (2020). Unconventional economics: methodology and methodics. 2nd Completed and updated edition. TSU Publishing. (in Georgian). 